\title{
DÜBLIN
}

Technological University Dublin

ARROW@TU Dublin

\section{Optimising Design Parameters of Enzyme-Channelling Biosensors}

\author{
Dana Mackey \\ Technological University Dublin, dana.mackey@tudublin.ie \\ Tony Killard \\ Dublin City University
}

Follow this and additional works at: https://arrow.tudublin.ie/scschphycon

Part of the Organic Chemistry Commons, and the Partial Differential Equations Commons

\section{Recommended Citation}

Mackey, D., \& Killard, T. (2008). Optimising Design Parameters of Enzyme-Channelling Biosensors. Progress in Industrial Mathematics, at ECMI 2006, Series: Mathematics in Industry, vol. 12, pg. 853-857. doi:10.1007/978-3-540-71992-2_148

This Conference Paper is brought to you for free and open access by the School of Physics \& Clinical \& Optometric Science at ARROW@TU Dublin. It has been accepted for inclusion in Conference Papers by an authorized administrator of ARROW@TU Dublin. For more information, please contact arrow.admin@tudublin.ie, aisling.coyne@tudublin.ie,gerard.connolly@tudublin.ie.

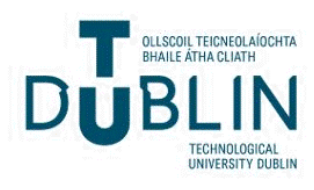




\title{
Optimising design parameters of enzyme-channelling biosensors
}

\author{
D. Mackey ${ }^{1}$ and A.J. Killard ${ }^{2}$ \\ 1 School of Mathematical Sciences, Dublin Institute of Technology, Ireland \\ Dana.Mackey@dit.ie \\ 2 National Centre for Sensor Research, Dublin City University, Ireland \\ Tony.Killard@dcu.ie
}

Summary. Two mathematical models for an electrochemical biosensor are proposed and compared with a view to determining the ratio of two immobilized enzymes which maximizes the amperometric signal amplitude.

Key words: Electrochemical biosensors, enzyme-substrate kinetics, diffusion equations, nonlinear boundary conditions, equilibrium analysis.

\section{Introduction}

Biosensors are devices in constant development due to their wide use in biomedical diagnostics and environmental monitoring. Of particular interest to developing electrochemical immunosensors are enzyme channelling systems, where two enzymes are brought in close proximity to an electrode surface thus facilitating the fast conversion of initial substrate to final product. Moreover, cascade schemes, where an enzyme is catalytically linked to another can produce signal amplification and therefore increase the device sensitivity.

This work investigates a biosensor employing the enzymes glucose oxidase (GOX) and horseradish peroxidase (HRP), immobilized on an electrode modified with a conducting polymer. After the immobilisation, the electrode is inserted in a flow-cell for an amperometric flow-injection analysis where glucose solutions at different concentrations are passed over the electrode and the signals recorded (see [KZZ99]). A mathematical model was proposed in [MKA07] and numerical simulations were carried out in order to determine the ratio of the two enzymes which maximizes current amplitude. In this paper, the optimal ratio of HRP and GOX, $\xi_{\max }$, is further investigated as a function of the kinetic rate constants and two different parameter régimes are identified, characterized by different qualitative behaviour of $\xi_{\text {max }}$. A simplified model is also introduced, which allows for an explicit formula for $\xi_{\max }$ to be derived and compared with the numerical simulations of the previous model. 


\section{Spatially extended model}

The flow effects are not explicitly modelled and the existence of the convective zone (where the glucose concentration is constant) is only reflected in the boundary conditions imposed at the top of the diffusion layer. The immobilized enzymes form a monolayer so all reactions can be assumed to take place at the lower boundary of the diffusion domain. The equations are onedimensional, where the variable $x$ measures the distance from the electrode.

A cascade reaction takes place at the electrode. Glucose oxidase catalyses the oxidation of glucose to gluconic acid, with production of hydrogen peroxide $\left(\mathrm{H}_{2} \mathrm{O}_{2}\right)$. HRP is oxidised by $\mathrm{H}_{2} \mathrm{O}_{2}$ and then subsequently reduced by electrons provided by the electrode. We model these reactions by a standard Michaelis-Menten kinetics scheme, (1), and we use the notation $E_{1}(t)=$ first enzyme (GOX) concentration, $E_{2}(t)=$ second enzyme (HRP) concentration, $S_{1}(x, t)=$ first substrate (glucose), $S_{2}(x, t)=$ second substrate $\left(\mathrm{H}_{2} \mathrm{O}_{2}\right)$, $C_{1}(t)=$ first complex, $C_{2}(t)=$ second complex, $P(x, t)=$ final product,

$$
E_{1}+S_{1} \underset{k_{-1}}{\stackrel{k_{1}}{\rightleftarrows}} C_{1} \stackrel{k_{2}}{\longrightarrow} E_{1}+S_{2}, \quad E_{2}+S_{2} \underset{k_{-3}}{\stackrel{k_{3}}{\rightleftarrows}} C_{2} \stackrel{k_{4}}{\longrightarrow} E_{2}+P .
$$

We now write down the differential equations governing the behaviour of the relevant chemical species. The two substrates, glucose and hydrogen peroxide are free to diffuse throughout the domain, hence

$$
\begin{gathered}
\frac{\partial S_{i}}{\partial t}=D_{i} \frac{\partial^{2} S_{i}}{\partial x^{2}}, \quad i=1,2, \quad 0 \leq x \leq L, \quad t \geq 0 \\
S_{1}(L, t)=S_{0} ; \quad S_{2}(L, t)=0,
\end{gathered}
$$

and the following boundary conditions hold on $x=0$

$$
\begin{gathered}
D_{1} \frac{\partial S_{1}}{\partial x}(0, t)=k_{1} E_{1} S_{1}-k_{-1} C_{1}, \\
D_{2} \frac{\partial S_{2}}{\partial x}(0, t)=k_{3} E_{2} S_{2}-\left(k_{2}+k_{-3}\right) C_{1}, \\
\frac{d E_{1}}{d t}=-k_{1} E_{1} S_{1}+\left(k_{-1}+k_{2}\right) C_{1}, \quad \frac{d E_{2}}{d t}=-k_{3} E_{2} S_{2}+\left(k_{4}+k_{-3}\right) C_{2}, \\
\frac{d C_{1}}{d t}=k_{1} E_{1} S_{1}-\left(k_{2}+k_{-1}\right) C_{1}, \quad \frac{d C_{2}}{d t}=k_{3} E_{2} S_{2}-\left(k_{4}+k_{-3}\right) C_{2}, \\
\frac{d P}{d t}=k_{4} C_{2} .
\end{gathered}
$$

The initial conditions are

$$
\begin{gathered}
S_{1}(x, 0)=S_{0}(x), \quad S_{2}(x, 0)=0, \quad P(x, 0)=0, \\
E_{1}(0)=\frac{\xi e}{1+\xi}, \quad E_{2}(0)=\frac{e}{1+\xi}, \quad C_{1}(0)=0, \quad C_{2}(0)=0,
\end{gathered}
$$


where $e$ is the total amount of enzyme present on the electrode and $S_{0}(x)=S_{0}$ if $x=L$ and 0 otherwise. The purpose of this study is to determine $\xi$, the ratio of GOX to HRP on the electrode, which maximizes the signal at the electrode. The measured current is given by the electron transfer rate, which can be assumed proportional to the rate of formation of product $P$ from (8).

\section{Simplified model}

In order to obtain an analytical expression for the dependence of the optimal enzyme ratio on other system parameters, we consider a simplified model which focuses on the kinetic surface processes, while neglecting transport of chemical species to and from the electrode. The main limitation in this case is failing to model the possibility of $\mathrm{H}_{2} \mathrm{O}_{2}$ to diffuse away from the electrode therefore assuming that all the product from the first reaction is readily available for the second.

With the assumption that the concentration of glucose is maintained constant at the reaction point, $S_{1}(t)=S_{0}$ for all $t \geq 0$, the model in the previous section now reduces to the following set of ordinary differential equations

$$
\begin{aligned}
& \frac{d C_{1}}{d t}=-\left(k_{1} S_{0}+k_{-1}+k_{2}\right) C_{1}+\frac{\xi e k_{1}}{1+\xi} S_{0} \\
& \frac{d C_{2}}{d t}=\frac{e k_{3}}{1+\xi} S_{2}-k_{3} S_{2} C_{2}-\left(k_{-3}+k_{4}\right) C_{2} \\
& \frac{d S_{2}}{d t}=k_{2} C_{1}+k_{-3} C_{2}-\frac{e k_{3}}{1+\xi} S_{2}+k_{3} S_{2} C_{2},
\end{aligned}
$$

with the initial conditions $C_{1}(0)=C_{2}(0)=S_{2}(0)=0$. There is a unique equilibrium point at $\left(C_{1}^{*}, C_{2}^{*}, S_{2}^{*}\right)$, where

$$
\begin{gathered}
C_{1}^{*}=\frac{\xi e}{1+\xi} \frac{k_{1} S_{0}}{k_{1} S_{0}+k_{-1}+k_{2}}, \quad C_{2}^{*}=\frac{\xi e}{1+\xi} \frac{k_{1} k_{2} S_{0}}{k_{4}\left(k_{1} S_{0}+k_{-1}+k_{2}\right)}, \\
S_{2}^{*}=\frac{\xi k_{1} k_{2}\left(k_{-3}+k_{4}\right) S_{0}}{\left(k_{1} k_{4} S_{0}+k_{-1} k_{4}+k_{2} k_{4}-\xi k_{1} k_{2} S_{0}\right) k_{3}} .
\end{gathered}
$$

The necessary condition for this equilibrium to be stable and positive is

$$
\xi k_{1} k_{2} S_{0}<k_{4}\left(k_{1} S_{0}+k_{-1}+k_{2}\right)
$$

and so, the value of $\xi$ which yields the highest $C_{2}^{*}$ value is

$$
\xi_{\max }=\frac{k_{4}}{k_{2}}\left(1+\frac{K_{M}^{1}}{S_{0}}\right)
$$

where $K_{M}^{1}=\left(k_{-1}+k_{2}\right) / k_{1}$ is the Michaelis constant of the first reaction. Hence, the simplified model shows that the optimal GOX:HRP ratio depends on the ratio of the turnover rates for the two consecutive reactions, as well as the number $1+K_{M}^{1} / S_{0}$ (the factor by which the velocity of the first reaction differs from its maximal velocity). 


\section{Numerical simulations and discussions}

Numerical simulations were carried out to establish how different kinetic parameters affect the current magnitude and optimal enzyme ratio. The system of equations (2)-(9) were integrated numerically using a standard finite difference method. In Fig. 1 we plot the steady state values of $\frac{d P}{d t}$, a measure of the amperometric signal, for different values of the molar ratio $\xi$, reaction speed ratio $k_{4} / k_{2}$ and various orders of magnitude of $K_{M}^{1} / S_{0}$. The optimal GOX:HRP ratio, $\xi_{\max }$, is then plotted in Fig. 2 as a function of $k_{4} / k_{2}$.
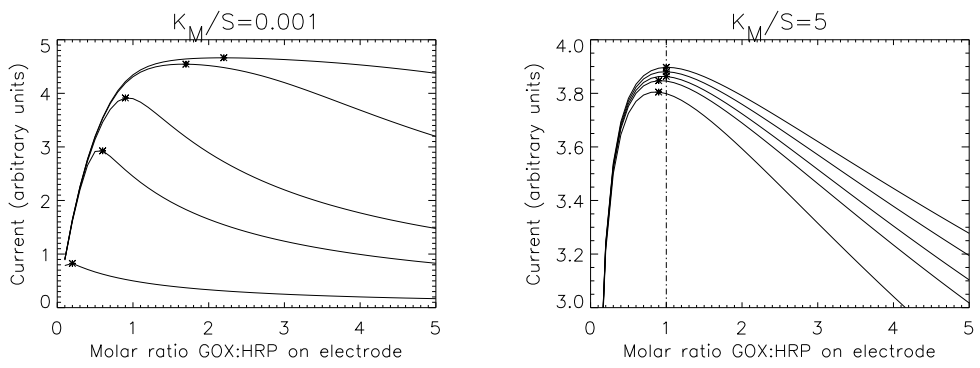

Fig. 1. Dependence of current on $\xi$ (electrode GOX:HRP ratio). From bottom to top, the curves correspond to $k_{4} / k_{2}=0.1,0.5,0.9,2.1,4.1$ for $K_{M}^{1} / S_{0}=0.001$ and $k_{4} / k_{2}=0.1,0.18,0.26,0.5,4$ for $K_{M}^{1} / S_{0}=5$.
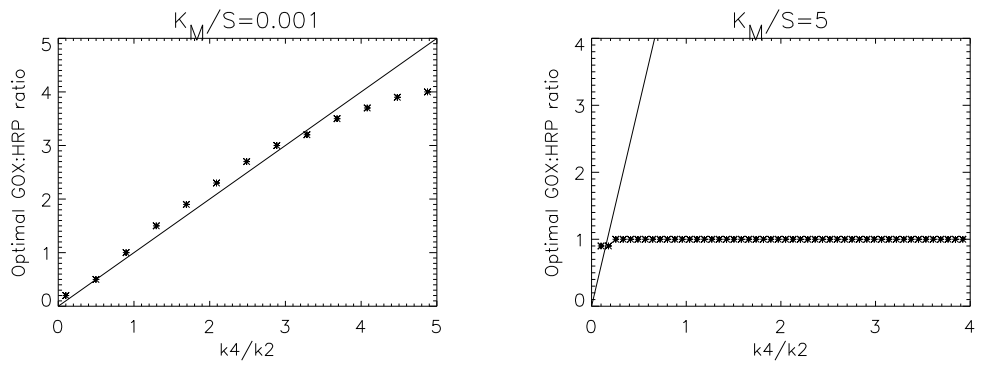

Fig. 2. Optimal GOX:HRP ratio as a function of $k_{4} / k_{2}$. Comparison of simplified model (straight line) and spatially extended model (curve)

When $K_{M}^{1} / S_{0} \ll 1$ (see Fig. 1(a)), the signal amplitude will increase with $\xi$, reach a maximum and then decrease. This is due to the fact that when the concentration of GOX increases, more $\mathrm{H}_{2} \mathrm{O}_{2}$ is produced. However, with increased production of hydrogen peroxide (as well as the reduced amount 
of HRP at higher values of $\xi$ ), there is a point where diffusion effects will dominate the second reaction and the resulting signal will decrease. This decay is faster when the second reaction is slow (small $k_{4}$ ) which explains why the lower curves in Fig. 1(a) are steeper than the higher ones. As a consequence, for high $k_{4}$, the optimal enzyme ratio is less relevant and the signal is not very sensitive to the concentrations of immobilized enzymes. For large $K_{M}^{1} / S_{0}$ in Fig. 1(b) the optimal $\xi$ value seems to be the same for most values of $k_{4}$. This can be explained by noting that since GOX is already idle, due to a relatively low amount of substrate, increasing its concentration will not result in increased production of hydrogen peroxide and will therefore not improve the efficiency of the system, regardless of how fast the second reaction is.

The main conclusion of these numerical simulations is the existence of two parameter régimes, $K_{M}^{1} / S_{0} \ll 1$ and $K_{M}^{1} / S_{0}=O(1)$, characterized by different qualitative behaviour of the current amplitude and optimal enzyme ratio, $\xi_{\max }$, as functions of the kinetic system variables. Moreover, for $K_{M}^{1} / S_{0} \ll 1$, there is good agreement between the one-point model and the spatially extended model, as the optimal ratio increases almost linearly with $k_{4} / k_{2}$. The slight divergence of results observed in Fig. 2(a) is only apparent since, for high values of $k_{4} / k_{2}$ it is more appropriate to speak of optimal $\xi$ intervals, rather than values. A rigorous asymptotic study of these parameter regions will be published separately.

Mathematical modelling is an excellent tool for biosensor design as it provides a theoretical framework through which to explore all the variables of a system without immediate recourse to experiment. For example, the high substrate concentration régime, $K_{M}^{1} / S_{0} \ll 1$, is more difficult to achieve experimentally due to limitations imposed by the physical solubility of glucose. In addition, our model can also assist in establishing values for constants that are difficult to establish experimentally. For instance, although an enzyme may possess a known ideal kinetic rate constant from solution-phase studies, this may change significantly when the enzyme is deposited on a surface.

\section{References}

[KZZ99] A.J. Killard, S. Zhang, H. Zhao, R. John, E.I. Iwuoha, and M.R. Smyth. Development of an electrochemical flow-injection immunoassay (FIIA) for the real-time monitoring of biospecific interactions. Anal Chim Acta, 400, 109-119 (1999).

[MKA07] D. Mackey, A.J. Killard, A. Ambrosi, and M.R. Smyth. Optimizing the ratio of horseradish peroxidase and glucose oxidase on a bienzyme electrode: comparison of a theoretical and experimental approach. Sensors and Actuators B: Chemical, 122, 395-402 (2007). 